\title{
Novel Sampling Clock Offset Estimation for DVB-T OFDM
}

\author{
Hou-Shin Chen Yumin Lee \\ Graduate Institute of Communication Eng. and \\ Department of Electrical Eng., \\ National Taiwan University, Taipei 10617, Taiwan
}

\begin{abstract}
Sampling clock offset estimation and compensation are important problems in an OFDM system. Sampling clock offset can cause a severe drift in symbol-timing, thus causing inter-carrier and inter-OFDM-symbol interference. In this paper, we propose a novel sampling clock offset estimator for OFDM systems that use scattered pilots. The proposed algorithm makes use of the received pilot phases and the least-squares algorithms. Simulation results show that when applied to the DFB-T standard, the performance of the proposed estimator is very accurate and robust against multipath fading and Doppler Spread.
\end{abstract}

\section{INTRODUCTION}

Terrestrial Digital Video Broadcasting (DVB-T) is a next-generation standard for wireless broadcast of MPEG-2 video [1]. In order to provide the high data rate required for video transmission, concatenated-coded orthogonal frequency division multiplexing (OFDM) has been adopted into DVB-T. In an OFDM system synchronization includes symbol timing estimation, carrier frequency offset estimation, and sampling clock offset estimation and compensation. Symbol-timing and frequency offset estimation for OFDM are widely discussed in the literature. However, relatively few results are available for the estimation of sampling clock offset. Sampling clock offset estimation and compensation are important in an OFDM system because sampling clock offset can cause a severe drift in symbol-timing, thus causing inter-carrier and inter-OFDM-symbol interference. The problem is especially severe when a large number of subcarriers is used. For example, in DVB-T with 2048 sub-carriers ( $2 \mathrm{~K}$ mode), if the sampling clock offset is 10 parts per million (ppm) of the sampling time duration, the resulting drift is about 77 samples per second. Therefore sampling clock offset synchronization is an important issue that needs to be solved for a practical OFDM system.

In this paper, we propose an algorithm using the DVB-T frequency-domain scattered pilot pattern shown in Fig.1 [1] for sampling clock offset estimation. Simulation results show that the proposed algorithm can reliably estimate the sampling clock offset from $10 \mathrm{ppm}$ to $200 \mathrm{ppm}$. The proposed algorithm can be used in AWGN or time-varying fading frequency-selective fading channels. Furthermore, channel estimation or decision feedback tracking loop [2][3] are not required, therefore the proposed algorithm has fairly low complexity. Simulation results show that the estimator proposed in this paper is very accurate and robust against multipath fading and Doppler spread.

\section{SySTEM SPECIFICATIONS}

Although DVB-T allows flexible choice of many transmission parameters, in this paper we assume that the
DVB-T transmitter uses OFDM with 16-level quadrature amplitude modulated (16 QAM) subcarriers. The number of subcarriers is $N=2048$ ( $2 \mathrm{~K}$ mode) and scattered pilots are present in the OFDM symbols as shown in Fig. 1. For $2 \mathrm{~K}$ mode, the useful part of an OFDM has a duration of $T_{U}$ $=298.6667 \mu \mathrm{s}$ (values for $6 \mathrm{MHz}$ channels). The length of the cyclic prefix $(\mathrm{CP})$ is assumed to be $0.125 T_{U}$, therefore the number of CP samples (chips) in an OFDM symbol is $\Delta=$ 256.

The OFDM signal is transmitted to the receiver via the wireless channel, which is assumed to be a multipath Rayleigh fading channel corrupted by additive white Gaussian noise (AWGN). The multipath fading channel is modeled using the modified Jakes' fading model [5] with a carrier frequency of $500 \mathrm{MHz}$. At the receiver, the received signal is first down-converted to the baseband, filtered, and sampled, and the three key steps of synchronization shown in Fig. 2 are next performed. The signal is first processed by the coarse symbol timing and frequency offset estimators. The frequency offset is next compensated, and the result is processed by the proposed sampling clock offset estimator.

\section{Channel Delay Estimation For OFDM}

Consider, for the time being, an OFDM transmission system with $N$ subcarriers operating over an additive white Gaussian noise (AWGN) channel with a constant delay of $\alpha$ samples, where $-0.5 \Delta \leq \alpha \leq 0.5 \Delta$, in which $\Delta$ is the number of samples in the cyclic prefix (CP). Further, assume that the two-sided power spectral density of AWGN is $N_{0} / 2$. The received frequency-domain subsymbol $Y_{i, k}$ is related to the transmitted subsymbol $X_{i, k}$ by [4]

$$
Y_{i, k}=X_{i, k} e^{-j 2 \pi k \alpha / N}+n_{i, k}
$$

where $i$ and $k$ are the OFDM symbol and subcarrier indices, respectively, and $n_{i, k}$ is the additive Gaussian noise. Assuming that in the $i$-th OFDM symbol, pilot subsymbols are available at subcarriers $k_{1} \ldots k_{P}$ where $P$ is the number of pilot subsymbols, an estimate of $\alpha$ can be obtained as a function of $\mathbf{Y}_{i} \equiv\left\lfloor Y_{i, k_{1}} \cdots Y_{i, k_{P}}\right\rfloor$ and $\mathbf{X}_{i} \equiv\left\lfloor X_{i, k_{1}} \cdots X_{i, k_{P}}\right\rfloor$. The estimator is given by

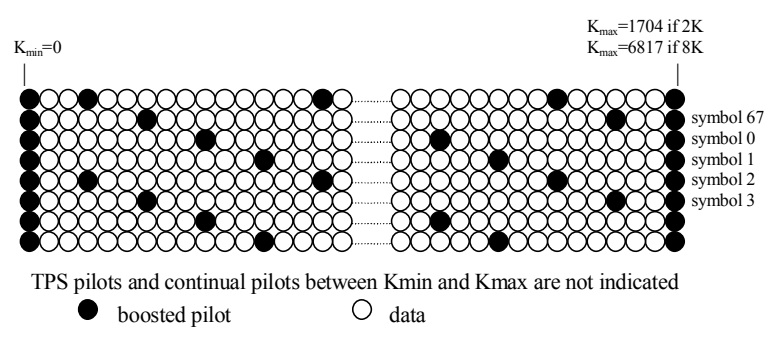

Fig.1 The pilot insertion pattern of DVB-T system. 


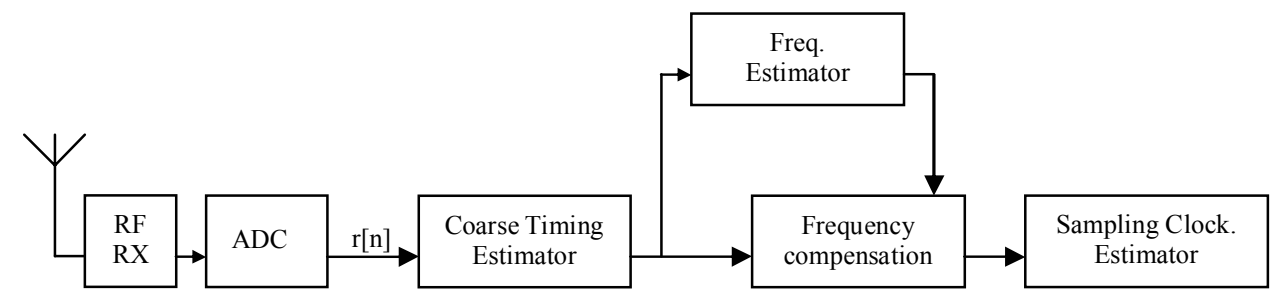

Fig. 2. Block diagram of the synchronization.

$$
\hat{\boldsymbol{\alpha}}\left(\mathbf{Y}_{i} ; \mathbf{X}_{i}\right) \equiv-\frac{N}{2 \pi} \times \frac{P \sum_{j=1}^{P} k_{j} P_{i, k_{j}}-\left(\sum_{j=1}^{P} k_{j}\right)\left(\sum_{j=1}^{P} P_{i, k_{j}}\right)}{P \sum_{j=1}^{P} k_{j}^{2}-\left(\sum_{j=1}^{P} k_{j}\right)^{2}}
$$

where

$$
P_{i, k}=p_{i, k}-n \pi,
$$

in which

$$
p_{i, k}=\tan ^{-1}\left\{\frac{\operatorname{Im}\left[\frac{Y_{i, k}}{X_{i, k}}\right]}{\operatorname{Re}\left[\frac{Y_{i, k}}{X_{i, k}}\right]}\right\}
$$

and $n$ is an integer that satisfies

$$
(n-0.5) \pi<p_{i, k}-p_{i, k-1}<(n+0.5) \pi \text {. }
$$

Note that the quantity in (2) is the slope of the least-squares (LS) best-fit-line of $P_{i, k}$ against $k$, where $P_{i, k}$ defined in (3) is an estimate of $-2 \pi k \alpha / N$ using $Y_{i, k}$ as observation and $X_{i, k}$ as reference.

Assuming that there is no sampling clock offset, the received pilot subsymbol in (1) can be re-written as

$$
Y_{i, k} \equiv X e^{j \theta_{k}}=A e^{-j 2 \pi \alpha k / N}+B e^{j \Phi}
$$

where $A=\sqrt{E\left[\left|X_{i, k}\right|^{2}\right]}, B$ is a Rayleigh distributed random variable with mean-square $N_{0}$, and $\Phi$ is random variable that is independent of $B$ and uniformly distributed in $[0,2 \pi]$. Note that in (6) we have omitted time indices whenever appropriate. From (6), we have

$$
\begin{gathered}
\theta_{k}=\tan ^{-1}\left(\frac{A \sin (-2 \pi \alpha k / N)+B \sin \Phi}{A \cos (-2 \pi \alpha k / N)+B \cos \Phi}\right) \\
=\tan ^{-1}\left[\tan (-2 \pi \alpha k / N)+\frac{\sin (2 \pi \alpha k / N+\Phi)}{(A / B) \cos ^{2}(2 \pi \alpha k / N)+\cos (2 \pi \alpha k / N) \cos \Phi}\right]
\end{gathered}
$$

Using a first approximation, (7) becomes

$$
\begin{aligned}
& \theta_{k}=-2 \pi \alpha k+\frac{1}{1+\tan ^{2}(2 \pi \alpha k / N)} \times \\
& \frac{B \sin (2 \pi \alpha k / N+\Phi)}{A \cos ^{2}(2 \pi \alpha k / N)+B \cos (2 \pi \alpha k / N) \cos \Phi}
\end{aligned}
$$

Defining the phase estimation error caused by AWGN as

$$
\begin{aligned}
& e_{k}=\frac{1}{1+\tan ^{2}(2 \pi \alpha k / N)} \times \\
& \frac{B \sin (2 \pi \alpha k / N+\Phi)}{A \cos ^{2}(2 \pi \alpha k / N)+B \cos (2 \pi \alpha k / N) \cos \Phi},
\end{aligned}
$$

the mean and variance of the estimator in (2) can be computed. Example numerical values are shown in Table 1 for $\alpha=20$ and $N=2048$. It can be seen from Table 1 that the estimator in (2) is approximately unbiased and has very small variance even at low signal-to-noise ratio (SNR) when there is no sampling clock offset.

In the presence of sampling clock offset, the received pilot sub-symbol can be approximated by [2]

$$
Y_{i, k}=e^{j\left[2 \pi \frac{k}{N}\left(\beta \frac{T_{s}}{T} k+\alpha\right)\right]} \operatorname{sinc}(n \beta) X_{i, k}+n_{i, k}+n_{i, k}^{\beta},
$$

where $\operatorname{sinc}(x)=\sin (\pi x) / \pi x, n_{i, k}$ is the same additive Gaussian noise as (1), $\beta$ is the sampling clock offset normalized by the sampling period of the transmitter (to be formally defined in the next section), and $n_{i, k}^{\beta}$ is additional noise caused by sampling clock offset caused with variance [2] given by

$$
\operatorname{Var}\left[n_{i, k}^{\beta}\right] \approx \frac{\pi^{2}}{3}(k \beta)^{2} \text {. }
$$

Note that $k \beta<<1$ in most practical cases, thus $\operatorname{sinc}(k \beta) \approx 1$ and $n_{i, k}^{\beta} \approx 0$. In this case the effect of sampling clock offset can be approximated simply as an additional phase rotation. It can therefore be argued that in the AWGN channel, the estimator in (2) is still accurate even in the presence of sampling clock offset.

\section{Proposed Sampling Clock Offset Estimator}

We next consider the estimation of sampling clock offset in a multipath fading environment. Denoting the sampling interval in the receiver as $T^{\prime}$, the normalized sampling clock offset is defined as

$$
\beta=\frac{T^{\prime}-T}{T}
$$

where $T$ is the sample-period of the transmitted OFDM signal. The effect of sampling clock offset can be roughly treated as an additional channel delay that varies with the OFDM symbol index. Mathematically, with sampling clock offset, the $i$-th OFDM symbol roughly experiences a delay (in samples) of

$$
\alpha_{i}=\alpha_{0}+\beta(N+\Delta) i
$$

Table 1 Mean and standard deviation of estimation error of (2)

\begin{tabular}{|c|c|c|c|c|}
\hline$A^{2} / N_{0}$ & $5 \mathrm{~dB}$ & $10 \mathrm{~dB}$ & $15 \mathrm{~dB}$ & $20 \mathrm{~dB}$ \\
\hline $\begin{array}{c}\text { mean } \\
\text { (samples) }\end{array}$ & $-9.334 \mathrm{e} \times 10^{-4}$ & $-1.104 \times 10^{-4}$ & $\approx 0$ & $\approx 0$ \\
\hline $\begin{array}{c}\text { Standard } \\
\text { deviation } \\
\text { (samples) }\end{array}$ & $9.267 \times 10^{-2}$ & $2.771 \times 10^{-2}$ & $1.273 \times 10^{-2}$ & $2 \times 10^{-3}$ \\
\hline
\end{tabular}


where $\alpha_{0}$ is the initial delay experienced by the 0 -th OFDM symbol. Since the estimator in (2) is relatively unaffected by sampling clock offset as previously mentioned, we can therefore build upon the estimator in (2) and obtain a new estimation algorithm for $\beta$. The key concept is to estimate $\alpha_{i}$ in several OFDM symbols, and compute the slope of the LS best-fit-line of these timing estimates against the OFDM symbol index $i$. In doing so, special measures are necessary to account for the random phase rotation introduced by the multipath fading channel so that the resulting estimate is robust against multipath fading. The proposed algorithm is as follows:

Step 1: Use (2) to compute $\hat{\alpha}_{i, j}=\hat{\alpha}\left(\mathbf{Y}_{i+4 j} ; \mathbf{Y}_{i}\right), i=$ $0 \ldots F-1, j=1 \ldots M$.

Step 2: Compute

$$
\hat{\beta}_{i}=\frac{1}{4(N+\boldsymbol{\Delta})} \times \frac{M \sum_{j=1}^{M} j \hat{\alpha}_{i, j}-\left(\sum_{j=1}^{M} j\right)\left(\sum_{j=1}^{M} \hat{\alpha}_{i, j}\right)}{M \sum_{j=1}^{M} j^{2}-\left(\sum_{j=1}^{M} j\right)^{2}}
$$

for $i=0 \ldots F-1$.

Step 3: Obtain a histogram of $\hat{\beta}_{0} \cdots \hat{\beta}_{F-1}$. The sampling clock offset estimate $\hat{\beta}$ is the mode (value with maximum occurring frequency) of the histogram.

Note that Step 1 estimates the delay experienced by the $(i+4 j)$-th OFDM symbol using $\mathbf{Y}_{i+4 j}$ as observation and $\mathbf{Y}_{i}$ as reference. In other words, at each pilot subcarrier $k$, the phase of $Y_{i+4 j, k} / Y_{i, k}$ is computed and substituted into (2). This is because in DVB-T, the pilot patterns in the $i$-th and $(i+4 j)$-th OFDM symbols are the same for all $i$ and $j$. Using $\mathbf{Y}_{i}$ instead of $\mathbf{X}_{i+4 j}$ as reference accounts for the random phase rotation introduced by the multipath fading channel and provides a noncoherent estimate of the delay experienced by the $(i+4 j)$-th OFDM symbol. In step $2, \hat{\beta}_{i}$ in (14) is the slope of the LS best-fit-line of $\hat{\alpha}_{i, j}$ against $j$, and is an estimate for $\beta$ based on the observations $\mathbf{Y}_{i+4 j}, j=$ $0 \ldots M$. Note that the estimation accuracy of $\hat{\beta}_{i}$ improves as $M$ increases. However, when the channel Doppler-spread is large, a small $M$ is desirable. Therefore the choice of $M$ provides a trade-off between estimation accuracy and robustness against Doppler-spread. Finally,

Step 3 provides the final estimate $\hat{\beta}$ from the noisy estimates $\hat{\beta}_{0} \cdots \hat{\beta}_{F-1}$. The method described in Step 3 is chosen empirically based on some preliminary simulations. Other approaches, e.g., taking the average of $\hat{\beta}_{0} \cdots \hat{\beta}_{F-1}$, are also possible. However, the method described in Step 3 seems to yield the best results.

\section{SimplificATIONS FOR THE AWGN CHANNEL}

The proposed sampling clock offset estimation algorithm can be greatly simplified when the OFDM system is operating in an AWGN channel. First, in Step 1 we can simply compute the phases of the received scattered pilots to obtain $\hat{\alpha}_{i, j}$ without worrying about random phase rotations introduced by the multipath fading channel. Second, in Step 2, we can set $F=1$, thus eliminating the need for Step 3 . The resulting simplified algorithm for the AWGN channel is as follows:

Step 1: Use (2) to compute $\hat{\alpha}_{i, j}=\hat{\alpha}\left(\mathbf{Y}_{i+4 j} ; \mathbf{X}_{i}\right), i=0, j$ $=1 \ldots M$.

Step 2: Compute

$$
\hat{\beta}_{i}=\frac{1}{4(N+\Delta)} \times \frac{M \sum_{j=1}^{M} j \hat{\alpha}_{i, j}-\left(\sum_{j=1}^{M} j\right)\left(\sum_{j=1}^{M} \hat{\alpha}_{i, j}\right)}{M \sum_{j=1}^{M} j^{2}-\left(\sum_{j=1}^{M} j\right)^{2}}
$$

for $i=0$.

Accurate estimation is achievable as long as $M$ is sufficiently large. Due to diminishing returns, when $M$ is sufficiently large only a marginal performance improvement is achievable by further increasing $M$.

\section{Symbol Timing And Frequency Offset ESTIMATION}

Although the focus of this paper is sampling clock offset estimation, symbol-timing and frequency-offset estimation are also performed in the computer simulations. The algorithms for symbol timing and frequency offset estimation are described as follows :

\section{Symbol Timing Estimation [6]}

Symbol timing estimation is accomplished by exploiting the cyclic nature of OFDM symbols. We define the autocorrelation function of the received time-domain OFDM signal as

$$
x[n]=\sum_{j=0}^{\Delta-1} r[n-j] r^{*}[n-j-N]
$$

and compute an average autocorrelation function defined as

$$
\bar{x}[n] \equiv \frac{1}{L} \sum_{l=0}^{L-1} x[n-l(N+\Delta)]
$$

where $L$ is the number of OFDM symbols observed for symbol-timing estimation. Assuming that the received signal has been frame-synchronized, the optimal timing (sample index of the beginning of the first OFDM symbol) is given by

$$
n_{0} \equiv \arg \max _{0 \leq n<N_{i}}|\bar{x}[n]|-\delta,
$$

where $\delta$ is an integer margin introduced to to ensure that the constant delay $\alpha$ is within the range of $-0.5 \Delta \leq \alpha \leq 0.5 \Delta$.

\section{Frequency Offset Estimation and Compensation}

After symbol-timing is determined, the frequency offset is next estimated and compensated for using a three-step frequency synchronization algorithm. Assuming that the frequency offset between the transmitter and receiver oscillators is given by

$$
\Delta f=(K+b) \frac{1}{T_{U}},
$$

where $K$ is an integer and $-0.5 \leq b<0.5$. It can be easily verified that, when there is no noise, the phase difference between a sample in the cyclic prefix of a received OFDM 
symbol and the sample $T_{U}$ seconds later is roughly $-2 \pi b$. Therefore an estimate for $b$ is given by

$$
b_{0} \equiv \frac{-1}{2 \pi} \operatorname{Arg}\left(\bar{x}\left[n_{0}\right]\right),
$$

where $\operatorname{Arg}(x)$ is the phase angle (modulo $2 \pi$ ) of $x$. On the other hand, $K$ can be estimated by making use of the continual pilots defined in DVB-T. We define the average correlation coefficient as

$$
\rho\left(k_{0}\right) \equiv \frac{\left\langle R\left(j+1, k_{p}+k_{0}\right) R^{*}\left(j, k_{p}+k_{0}\right)\right\rangle}{\sqrt{\left\langle\left|R\left(j+1, k_{p}+k_{0}\right)\right|^{2}\right\rangle\left\langle\left|R\left(j, k_{p}+k_{0}\right)\right|^{2}\right\rangle}},
$$

where $\diamond$ denotes averaging over the continual pilot subcarrier index $k_{p}$. It can be easily argued that, when there is no noise, $\rho\left(k_{0}\right)$ is maximized when $k_{0}=K$. Therefore a reasonable estimate for $K$ is given by

$$
K_{0} \equiv \max _{k_{0}} \rho\left(k_{0}\right)
$$

The estimated frequency offset is then given by $\left(K_{0}+b_{0}\right) / T_{U}$ Hz. Preliminary simulations show that when $b \approx 0.5$, separately using (19) and (21) for frequency offset estimation may result in poor performance in the presence of noise. For example, if $K+b=2.45$ and $K_{0}=3$, then the error in $K_{0}$ cannot be completely compensated because $b_{0} \geq-0.5$. In order to solve this problem, in this paper the receiver frequency synchronization is done in three steps. In the first step, (19) is evaluated to obtain an estimate $b_{0}$. The received signal is then frequency-compensated by $b_{0} / T_{U} \mathrm{~Hz}$ to make the residual frequency offset approximately equal to a multiple of the carrier spacing. In the second step, (21) is evaluated to obtain an estimate $K_{0}$, and the received signal is frequency-compensated by $K_{0} / T_{U} \mathrm{~Hz}$. Finally, (19) is evaluated again to recover the remaining frequency offset.

\section{Simulation Results}

The sampling clock offset estimation algorithm proposed in this paper is evaluated using computer simulations. The transmitter is simulated according to the DVB-T standard. The wireless channel is modeled as Rayleigh multipath fading channel with exponential power-delay profile with RMS delay spread of $5.2 \mu \mathrm{s}$ and corrupted by AWGN. The carrier frequency offset is $2.5 / T_{U}$. At the receiver, the received signal is filtered and sampled at a rate of $1 / T^{\prime}$. Coarse symbol-timing recover and frequency-offset compensation is next performed. Sampling clock offset estimation is finally performed using the proposed method with $F=63$ and $M=2$ in the fading channel and $F=1$ and $M=30$ in AWGN channel.

The root-mean-square (RMS) residual sampling clock offset $^{1}$ is shown in Fig. 1 to Fig.3 for the AWGN, quasi-static frequency-selective, and time-varying frequency-selective fading channels as functions of $E_{\mathrm{s}} / N_{0}$ for different values of $\beta$, where $E_{s}$ is the average symbol energy per subcarrier. In Fig. 3 the maximum normalized Doppler-spread of the channel is $f_{m} T_{U}=0.015$, which corresponds to a vehicle speed of $100 \mathrm{~km} / \mathrm{h}$. It can be seen that for the frequency-selective fading channels, at high SNR the RMS residual sampling clock offset is less than $2 \mathrm{ppm}$ even when $\beta$ is as high as $200 \mathrm{ppm}$.

\footnotetext{
1 This is equal to the RMS sampling clock offset estimation error.
}

The channel bit error rate (BER) is plotted as functions of $E_{\mathrm{s}} / N_{0}$ in Fig. 4 for frequency-selective fading channels with $f_{m} T_{U}=0$ (quasi-static) and $f_{m} T_{U}=0.015$. The sampling clock offset is $\beta=50 \mathrm{ppm}$. In each case three receivers are simulated: 1) a receiver that estimates the sampling clock offset using the proposed approach and uses the sinc interpolation for compensation (labeled as "PROPOSED") ; 2) a receiver with perfect sampling clock offset compensation (labeled as "PERFECT"); and 3) a receiver (labeled as "NO SYNC") without sampling clock offset compensation in each frame (68 OFDM symbols). All receivers use the same symbol-timing and frequency offset estimation algorithms. It can be seen that, if uncompensated, a sampling clock offset of $50 \mathrm{ppm}$ causes significant degradation in BER. Furthermore, the proposed algorithm is very effective and achieves almost the same performance as the "perfect" case. Finally, the proposed algorithm is robust against Doppler-spread because it achieves roughly the same performance for both values of $f_{m} T_{U}$.

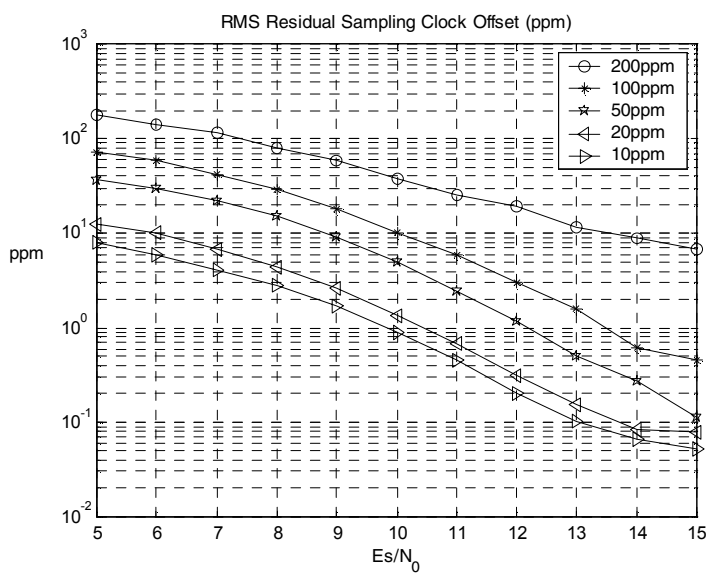

Fig. 1 RMS residual sampling clock offset (ppm) in AWGN channel.

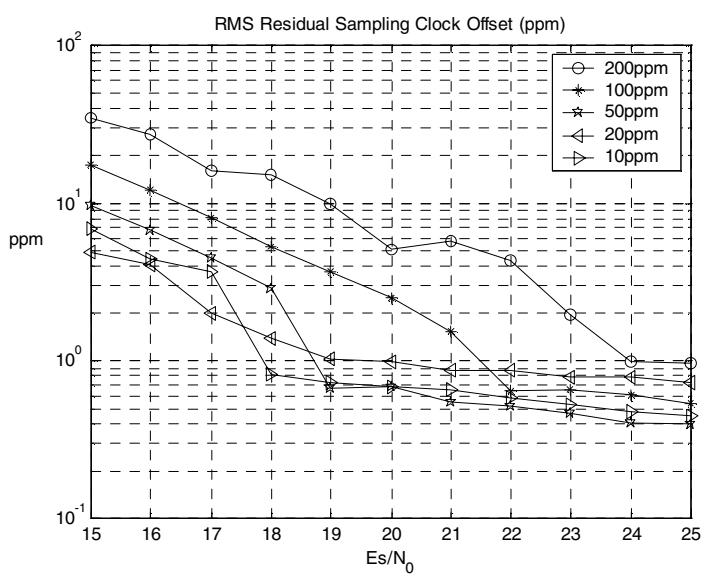

Fig. 2 RMS residual sampling clock offset (ppm) in a frequency selective fading channel without Doppler effect. 


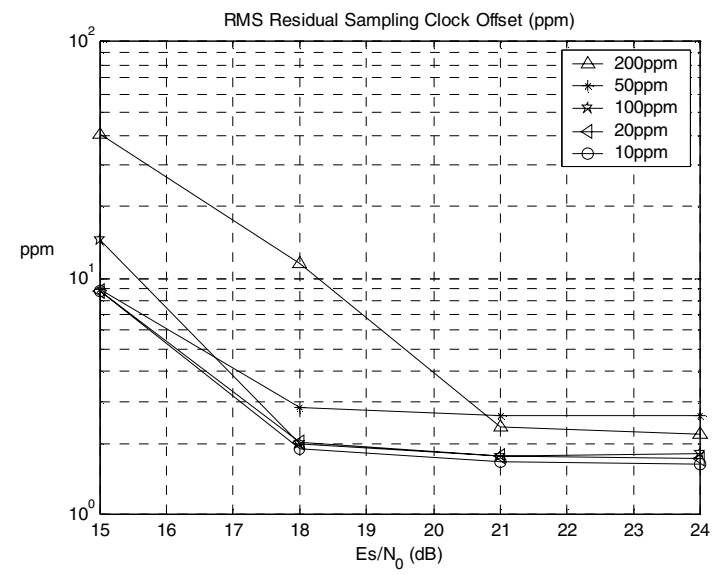

Fig. 3 RMS residual sampling clock offset (ppm) in a frequency selective fading channel with maximum Doppler-spread $f_{m} T_{U}=0.015$.

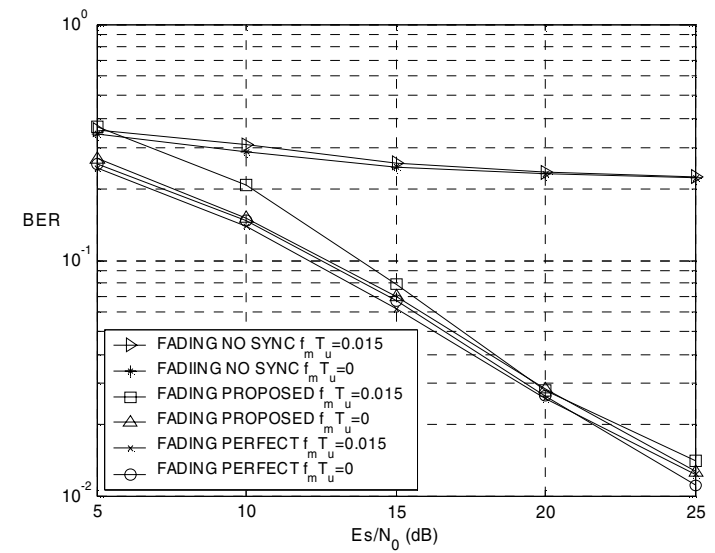

Fig. 4 BER of perfect sampling clock compensation, the proposed algorithm, and without sampling clock offset compensation in frequency selective fading channels.

\section{CONCLUSION}

In this paper, we propose a sampling clock offset estimation algorithm using the DVB-T frequency-domain scattered pilot pattern. The algorithm builds upon a least-squares estimator for channel delay and can be used in AWGN or time-varying fading frequency-selective fading channels. Channel estimation or decision feedback tracking loop [2][3] are not required, therefore the proposed algorithm has fairly low complexity. Simulation results show that the proposed algorithm the estimator proposed in this paper is very accurate and robust against multipath fading and Doppler spread, and can reliably estimate the sampling clock offset from $10 \mathrm{ppm}$ to $200 \mathrm{ppm}$.

\section{REFERENCES}

[1] ETSI, "Digital Video Broadcasting: Framing Structure, Channel coding, and Modulation for Digital Terrestrial Television", European Telecommunication Standard EN300744, Aug. 1997.

[2] Baoguo Yang, Zhengxin Ma, and Zhigang Cao, "ML-Oriented DA Sampling Clock Synchronization for OFDM Systems", International Conference on Communication Technology, 2000, Vol.1, pp. 781-784.

[3] Baoguo Yang, K. B. Letaief, Roger S. Cheng, and Zhigang Cao, "An Improved Combined Symbol and Sampling Clock Synchronization Method for OFDM Systems", IEEE Wireless Communication and Networking Conference, WCNC, 1999, Vol. 3, pp.1153-1157.

[4] Yong-Jung Kim, Dong-Seog Han and Ki-Bum Kim, “A New Fast Symbol Timing Recovery Algorithm for OFDM Systems", IEEE Transactions on Consumer Electronics, Vol.44, No.3, pp.1134-1141, August 1998.

[5] P. Dent, G.E. Bottomley, and T. Croft, Jakes fading model revisited," Electronics Letters, Vol.29, No.13, pp.1162-1163, June 1993.

[6] Richard van Nee, Ramjee Prasad, OFDM for Wireless Multimedia Communications, Artech house, 2000. 